\title{
On the Principal Eigencurve of the p-Laplacian: Stability Phenomena
}

\author{
Abdelouahed El Khalil, Said El Manouni, and Mohammed Ouanan
}

Abstract. We show that each point of the principal eigencurve of the nonlinear problem

$$
-\Delta_{p} u-\lambda m(x)|u|^{p-2} u=\mu|u|^{p-2} u \text { in } \Omega,
$$

is stable (continuous) with respect to the exponent $p$ varying in $(1, \infty)$; we also prove some convergence results of the principal eigenfunctions corresponding.

\section{Introduction}

In this paper we study the stability (continuity) of the two parameter nonlinear problem

$$
-\Delta_{p} u-\lambda m(x)|u|^{p-2} u=\mu|u|^{p-2} u \quad \text { in } \Omega
$$

with respect to the exponent $p$. The solution $u$ is scalar valued and required to belong to the Sobolev space $W_{0}^{1, p}(\Omega)$, where $\Omega$ is a bounded domain in the Euclidean space $\mathbb{R}^{N}$ and the real parameters $\lambda$ and $\mu$ play the role of eigenvalues. The weight $m \in$ $L^{\infty}(\Omega) \backslash\{0\}$ can change its sign. Here $1<p<\infty$ is considered as a variable exponent.

The nonlinear degenerate operator $\Delta_{p} u=\nabla \cdot\left(|\nabla u|^{p-2} \nabla u\right)$, is the so-called $p$-Laplacian, which is well known to mathematicians for being a prototype for monotone operators, see $[20,24]$. This operator has attracted growing interest, and occurs in a large variety of application in applied fields and many mathematical models of physical topics, according to values of the exponent $p$. For example, in the mathematical modelling of non-Newtonian fluids (dilatant fluids for $p>2$, pseudo-plastics for $1<p<2$ ) [4], reaction-diffusion problems, nonlinear elasticity and glaciology for $p=\frac{4}{3}$ [22], in power-lower materials [3], in nonlinear diffusion and filtration problems [23], in flow porous media, in petroleum extraction and torsional creep problems [10] and the thermal radiation of a hydrogen bomb [6]. Note that the $p$-Laplacian counterpart of Smagornsky's model corresponds to $p=3[25]$. For a discussion of some physical background, see [10]. For the linear case $(p=2)$, there is an extensive literature.

\footnotetext{
Received by the editors July 4, 2004; revised February 16, 2005.

AMS subject classification: 35P30, 35P60, 35J70.

Keywords: $p$-Laplacian with indefinite weight, principal eigencurve, principal eigenvalue, principal eigenfunction, stability.

(c) Canadian Mathematical Society 2006.
} 
Note that when $p=2$, we just get the usual Laplacian and we are back to a wellunderstood familiar linear equation

$$
\Delta u+\lambda m(x) u+\mu u=0
$$

appearing in connection with Schrödinger's equation [9].

The above shows the importance of studying the dependence of the principal eigenvalue of $\left(\mathcal{E}_{p}\right)$ with the exponent $p$. This is the main objective of the present paper. The difficulty is that the Sobolev spaces $W_{0}^{1, p}(\Omega)$ change with the exponent $p$. We overcome this obstacle by assuming that the underlying domain $\Omega$ has the so-called segment property, a sufficient regularity condition related to the geometry.

The problem $\left(\varepsilon_{p}\right)$ was considered by Hess and Kato [17] (for the linear case $p=2$ ) and by Binding and Huang [7], in a smooth bounded domain. It was also considered by various authors for the particular cases $\lambda=0$ or $\mu=0$. We cite the works of Anane [2], Azorero and Alonso [5], Otani and Theshima [21] and Lindqvist [18], just to mention a few authors. Note that the stability question is not considered in the above references.

In the particular case $\lambda=0$ in $\left(\varepsilon_{p}\right)$, the stability phenomena were studied essentially by Lindqvist in [19]. See also [14] for $\mu=0$ and where $\lambda$ plays the role of eigenvalue.

The present paper is partially motivated by recent works $[7,13,15]$. The simplicity of each point of the first eigencurve of $\left(\varepsilon_{p}\right)$ in a general bounded domain $\Omega$ was shown in [15]. Here the first (or principal) eigencurve of $\left(\varepsilon_{p}\right)$ means the graph of the numerical function $\mu_{1}: \lambda \rightarrow \mu_{1}(\lambda)$ from $\mathbb{R}$ to $\mathbb{R}$, where $\mu_{1}(\lambda)$, for fixed $\lambda$, denotes the principal eigenvalue of $\left(\varepsilon_{p}\right)$.

We now describe some of the results of this paper. To prove our main results, i.e., the stability of the problem $\left(\varepsilon_{p}\right)$ with respect to $p$, which varies continuously (Theorem 3.7 and Theorem 4.1), we were able to adapt some techniques of [19] to our problems with some modifications. However, our situation is bit more complicated due to the fact that the first eigenvalue of $\left(\varepsilon_{p}\right), \mu_{p}(\lambda)=\mu_{1}(\lambda)$ (indicating the dependance on $p$ ) can take negative values for some $\lambda \in \mathbb{R}$, because the weight $m$ is indefinite and can change its sign on $\Omega$. Lindqvist [19] proved that the stability of $\left(\varepsilon_{p}\right)$, for the particular case $\lambda=0$, may fail in an irregular domain, but it is true for a smooth domain. Our first result establishes that this result also holds true for a bounded domain such that $W^{1, p}(\Omega) \cap W_{0}^{1, p-\varepsilon}(\Omega)=W_{0}^{1, p}(\Omega)$, for some $\varepsilon>0$. So we include this equality (Theorem 2.2) when $\Omega$ is a bounded domain having the socalled segment property. This class of domains is fairly large. This property is needed here to guarantee the right boundary values of the limit function. This is all the more interesting when $p<N$ (because for $p \geq N$, any function in $W_{0}^{1, p}(\Omega)$ is continuous on $\bar{\Omega}$, by Sobolev embedding, and consequently the stability is required).

The other main objective of our paper is to study the convergence of the principal eigenfunctions in connection with the inequalities

$$
\lim _{s \rightarrow p_{-}} \mu_{s}(\lambda) \leq \mu_{p}(\lambda)=\lim _{s \rightarrow p_{+}} \mu_{s}(\lambda)
$$

proved in Corollary 3.5, by using a direct method based essentially on the variational 
characterization of corresponding principal eigenvalues. For $\lambda=0$ the states' inequalities are due to Lindqvist [19].

The outline of this paper is as follows. In Section 2, we establish some definitions, notations and basic properties. In Section 3, we first give some general stability results with respect to $p$ for each point of the first eigencurve of problem $\left(\varepsilon_{p}\right)$ and then we restrict ourselves to a bounded domain having the segment property. In Section 4 , we establish some convergence results of the principal eigenfunctions denoted $u_{p}(\lambda), p \in(1,+\infty)$, corresponding to $\mu_{p}(\lambda)$.

\section{Preliminaries, Notations and Definitions}

In defining the eigenpairs of $\left(\mathcal{E}_{p}\right)$ (in a given bounded domain $\Omega \subset \mathbb{R}^{N}$ ) we shall interpret the equation $\left(\varepsilon_{p}\right)$ in the weak sense. We say that $(\lambda, \mu) \in \mathbb{R} \times \mathbb{R}$ is an eigenpair of $\left(\varepsilon_{p}\right)$, if there exists a function $u \in W_{0}^{1, p}(\Omega) \backslash\{0\}$, such that the pair $((\lambda, \mu), u)$ satisfies $\left(\mathcal{E}_{p}\right)$ in the weak sense, i.e., such that

$$
\int_{\Omega}|\nabla u|^{p-2} \nabla u \nabla \varphi d x=\int_{\Omega}(\lambda m(x)+\mu)|u|^{p-2} u \varphi d x
$$

whenever $\varphi \in W_{0}^{1, p}(\Omega)$. The function $u$ is called an eigenfunction.

We define

$$
\mathrm{U}_{p}=\left\{u \in W_{0}^{1, p}(\Omega),\|u\|_{p}=1\right\} .
$$

For $\lambda \in \mathbb{R}$, let $\mu_{p}(\lambda)$ denote the first eigenvalue of $\left(\mathcal{E}_{p}\right)$. We recall from [15] that $\mu_{p}(\lambda)$ can be variationally characterized as

$$
\mu_{p}(\lambda)=\inf \left\{\frac{\|\nabla u\|_{p}^{p}-\lambda \int_{\Omega} m(x)|u|^{p} d x}{\|u\|_{p}^{p}} ; u \in W_{0}^{1, p}(\Omega) \backslash\{0\}\right\},
$$

that is,

$$
\mu_{p}(\lambda)=\min \left\{\|\nabla u\|_{p}^{p}-\lambda \int_{\Omega} m(x)|u|^{p} d x ; u \in W_{0}^{1, p}(\Omega),\|u\|_{p}=1\right\} .
$$

The graph of the function $\lambda \rightarrow \mu_{p}(\lambda)$ from $\mathbb{R}$ into $\mathbb{R}$ is called the first eigencurve of the $p$-Laplacian, which is also called the principal eigencurve of the $p$-Laplacian.

Throughout this paper, the first eigenfunctions are the eigenfunctions corresponding to eigenpair $\left(\lambda, \mu_{p}(\lambda)\right)$. And the principal eigenfunction, denoted $u_{p}(\lambda)$, is the first eigenfunction required to be positive and belongs to $U_{p}$. Hence, for any $\lambda \in \mathbb{R}$ we have

$$
\mu_{p}(\lambda)=\left\|\nabla u_{p}(\lambda)\right\|_{p}^{p}-\lambda \int_{\Omega} m(x)\left\{u_{p}(\lambda)\right\}^{p} d x
$$

We end this paragraph by recalling the following properties that can be found in [15]: 
(P1) $\mu_{p}(\lambda)$ can have negative values and $\mu_{p}(0)=\lambda_{1}$ (i.e., the first eigenvalue of the $p$-Laplacian );

(P2) for any $\lambda \in \mathbb{R}$, the first eigenfunctions are essentially unique for any bounded domain, i.e., they are merely constant multiples of each other;

(P3) for any $\lambda \in \mathbb{R}$, the first eigenfunctions have no zeros in the domain and they are the only eigenfunctions not changing sign;

(P4) for any $\lambda \in \mathbb{R}, \mu_{p}(\lambda)$ is the only eigenvalue of $\left(\varepsilon_{p}\right)$ having an eigenfunction not changing sign;

(P5) for any $\lambda \in \mathbb{R}, \mu_{p}(\lambda)$ is simple and isolated.

Now, we define a class of domains for which the boundary is sufficiently regular to guarantee that

$$
W^{1, p}(\Omega) \bigcap_{q<p} W_{0}^{1, q}(\Omega)=W_{0}^{1, p}(\Omega) .
$$

Definition 2.1 An open subset $\Omega$ of $\mathbb{R}^{N}$ is said to have the segment property if, given any $x \in \partial \Omega$, there exists an open set $G_{x}$ in $\mathbb{R}^{N}$ with $x \in G_{x}$, and a point $y_{x}$ of $\mathbb{R}^{N} \backslash\{0\}$ such that if $z \in \bar{\Omega} \cap G_{x}$ and $t \in(0,1)$, then $z+t y_{x} \in \Omega$.

This property allows us to push the support of a function $u$ in $\Omega$ via a translation. The following result is essential here.

Theorem $2.2([13,14]) \quad$ Let $\Omega$ be a bounded domain in $\mathbb{R}^{N}$ having the segment property. If $u \in W^{1, p}(\Omega) \cap W_{0}^{1, q}(\Omega)$ for some $q \in(1, p)$, then $u \in W_{0}^{1, p}(\Omega)$.

Remark 2.3 (i) If $\partial \Omega$ is of class $C^{1}$, then the proof is simple, since we can use the local carts, $c f$. [8]. (ii) The theorem holds if $\Omega$ is not necessarily bounded. It suffices to approximate $u$ arbitrarily closely in $W^{1, p}(\Omega)$ by functions with bounded supports. (iii) If $\Omega$ does not have the segment property, then the result of the theorem cannot hold. (iv) Note that a bounded domain $\Omega \subset \mathbb{R}^{N}$ has the segment property if and only if it is in the class $\mathcal{C}, c f$. [12]. This means that locally the boundary has the continuous equation $x_{N}=f\left(x_{1}, x_{2}, \ldots, x_{N-1}\right)$, after a notation of the coordinate axis. (v) We also note that $F\left(\Omega_{1}\right)$ has the segment property if $\Omega_{1}$ has this property and $F$ and $F^{-1}$ are Lipschitz. This result is a consequence of (iv).

Example 2.4 The set $\left\{x \in \mathbb{R}^{N} ; 0<|x|<1\right\}$ is a domain not having the segment property.

In what follows, we will need the next lemma which can be found in [15].

Lemma 2.5 For any $\lambda \in \mathbb{R}$ and for any bounded domain $\Omega$,

$$
\|u\|_{\infty} \leq 4^{N}\left(\left|\mu_{p}(\lambda)\right|+|\lambda|\|m\|_{\infty}\right)^{\frac{N}{p}}\|u\|_{1}
$$

for each $p \in(1, \infty)$ and for each eigenfunction $u$ associated to eigenpair $\left(\lambda, \mu_{p}(\lambda)\right)$. 


\section{Remark 2.6}

(i) Remark that if we take $u$ in $\mathrm{U}_{p}$, then Hölder's inequality yields

$$
\|u\|_{\infty} \leq 4^{N}\left(\left|\mu_{p}(\lambda)\right|+|\lambda|\|m\|_{\infty}\right)^{\frac{N}{p}}|\Omega|^{\frac{p-1}{p}},
$$

where $|\Omega|$ denotes the Lebesgue measure of $\Omega$.

(ii) From (2.6) it is clear that each eigenfunction of $\left(\mathcal{E}_{p}\right)$ belongs to $L^{\infty}(\Omega)$. By an advanced result in regularity theory, see [11], it follows that each eigenfunction is of class $C_{l o c}^{1, \alpha}$, for some $\alpha \in(0,1)$. Note that $\alpha$ depends on $\lambda$ and on the exponent $p$.

\section{Stability Results}

First, from (2.4) we deduce

$$
\left\|\nabla u_{p}(\lambda)\right\|_{p}^{p}=\mu_{p}(\lambda)+\lambda \int_{\Omega} m(x)\left\{u_{p}(\lambda)\right\}^{p} d x .
$$

Lemma 3.1 For any $\lambda \in \mathbb{R}$ and for any bounded range $I \subset(1, \infty)$, the sets $\left\{\mu_{p}(\lambda)\right.$; $p \in I\}$ and $\left\{\left\|\nabla u_{p}(\lambda)\right\|_{p}^{p} ; p \in I\right\}$ are uniformly bounded, that is,

$$
\max _{p \in I}\left\{\max \left(\left|\mu_{p}(\lambda)\right|,\left\|\nabla u_{p}(\lambda)\right\|_{p}^{p}\right)\right\}<\infty .
$$

Proof Let $u_{p}(0)$ be the principal eigenfunction associated to $\mu_{p}(0)=\lambda_{1}(p)$ (the first eigenvalue of $p$-Laplacian). Thus $u_{p}(0) \in \mathrm{U}_{p}, u(0)>0$ and

$$
\lambda_{1}(p)=k\left\|\nabla u_{p}(0)\right\|_{p}^{p}
$$

Hence $u_{p}(0)$ is admissible in the definition of $\mu_{p}(\lambda)$. It follows that

$$
\mu_{p}(\lambda) \leq \lambda_{1}(p)-\lambda \int_{\Omega} m(x)\left\{u_{p}(0)\right\}^{p} d x \leq \lambda_{1}(p)+|\lambda|\|m\|_{\infty} .
$$

Remark that $u_{p}(\lambda)$ is admissible in the definition of $\lambda_{1}(p)$. Therefore

$$
\lambda_{1}(p) \leq\left\|\nabla u_{p}(\lambda)\right\|_{p}^{p}
$$

Hence

$$
-\mu_{p}(\lambda) \leq \lambda_{1}(p)+|\lambda|\|m\|_{\infty}
$$

This and (3.2) yield

$$
\left|\mu_{p}(\lambda)\right| \leq \lambda_{1}(p)+|\lambda|\|m\|_{\infty} .
$$

On the other hand, from (2.4) we deduce that

$$
\left\|\nabla u_{p}(\lambda)\right\|_{p}^{p} \leq \lambda_{1}(p)+|\lambda|\|m\|_{\infty} .
$$

Using $[19,(2.5)]$, we can show that $\max _{p \in I} \lambda_{1}(p)$ is bounded by a finite constant. This fact, (3.4) and (3.5) achieve the proof. 
Remark 3.2 In a smooth domain one can use the continuity of the function $p \rightarrow$ $\lambda_{1}(p)$ to conclude that $\max _{p \in I} \lambda_{1}(p)$ is finite, see [19]. Then from (3.4) and (3.5) we deduce the result.

Proposition 3.3 For any $\lambda \in \mathbb{R}$ and for any bounded domain $\Omega$, we have

$$
\mu_{p}(\lambda) \leq \mu_{s}(\lambda)+\left[\left(\frac{s}{p}\right)^{p}\left(\lambda_{1}(s)\right)^{\frac{s}{p}-1}-1\right]\left\|\nabla u_{s}(\lambda)\right\|_{s}^{s},
$$

for any $s, p \in(1, \infty)$ such that $p<s$.

Proof The principal eigenfunctions $\left(u_{s}(\lambda)\right)_{s>p}$ associated to $\left(\mu_{s}(\lambda)\right)_{s>p}$ verify

$$
u_{s}(\lambda) \in \mathrm{U}_{s}, \quad u_{s}(\lambda)>0
$$

and

$$
\mu_{s}(\lambda)=\left\|\nabla u_{s}(\lambda)\right\|_{s}^{s}-\lambda \int_{\Omega} m(x)\left\{u_{s}(\lambda)\right\}^{s} d x .
$$

If we set $\varphi=\left\{u_{s}(\lambda)\right\}^{\frac{s}{p}}$, then $\varphi \in W_{0}^{1, p}(\Omega)$, because $p<s$. Thus $\varphi$ is admissible in the definition of $\mu_{p}(\lambda)$ and $\|\varphi\|_{p}=1$. Therefore

$$
\begin{aligned}
\mu_{p}(\lambda) & \leq\|\nabla \varphi\|_{p}^{p}-\lambda \int_{\Omega} m(x) \varphi^{p} d x \\
& =\left(\frac{s}{p}\right)^{p} \int_{\Omega}\left\{u_{s}(\lambda)\right\}^{s-p}\left|\nabla u_{s}(\lambda)\right|^{p} d x-\lambda \int_{\Omega} m(x)\left\{u_{s}(\lambda)\right\}^{s} d x,
\end{aligned}
$$

because $\varphi^{p}=\left\{u_{s}(\lambda)\right\}^{s}$ and $\nabla \varphi=\frac{s}{p}\left\{u_{s}(\lambda)\right\}^{\frac{s}{p}-1} \nabla u_{s}(\lambda)$. Hölder's inequality yields

$\mu_{p}(\lambda) \leq\left(\frac{s}{p}\right)^{p}\left(\int_{\Omega}\left\{u_{s}(\lambda)\right\}^{s} d x\right)^{1-\frac{p}{s}}\left(\int_{\Omega}\left|\nabla u_{s}(\lambda)\right|_{s} d x\right)^{\frac{p}{s}}-\lambda \int_{\Omega} m(x)\left\{u_{s}(\lambda)\right\}^{s} d x$.

Hence

$$
\mu_{p}(\lambda) \leq\left(\frac{s}{p}\right)^{p}\left(\int_{\Omega}\left|\nabla u_{s}(\lambda)\right|^{s} d x\right)^{\frac{p}{s}}-\lambda \int_{\Omega} m(x)\left\{u_{s}(\lambda)\right\}^{s} d x,
$$

because $u_{s}(\lambda) \in \mathrm{U}_{s}$. Since for $\frac{p}{s}<1$,

$$
\left(\int_{\Omega}\left|\nabla u_{s}(\lambda)\right|^{s} d x\right)^{\frac{p}{s}} \leq\left(\int_{\Omega}\left|\nabla u_{s}(\lambda)\right|^{s} d x\right)^{\frac{p}{s}-1}\left(\int_{\Omega}\left|\nabla u_{s}(\lambda)\right|^{s} d x\right)
$$

and

$$
\left(\int_{\Omega}\left|\nabla u_{s}(\lambda)\right|^{s} d x\right)^{\frac{p}{s}-1} \leq\left(\lambda_{1}(s)\right)^{\frac{p}{s}-1}
$$

we obtain

$$
\mu_{p}(\lambda) \leq\left(\frac{s}{p}\right)^{p}\left(\lambda_{1}(s)\right)^{\frac{p}{s}-1}\left(\int_{\Omega}\left|\nabla u_{s}(\lambda)\right|^{s} d x\right)-\lambda \int_{\Omega} m(x)\left\{u_{s}(\lambda)\right\}^{s} d x .
$$

The desired inequality follows from (3.8) and (3.7). 
Remark 3.4 If $\lambda=0$, then $\mu_{p}(0)=\lambda_{1}(p)$ and $\mu_{s}(0)=\lambda_{1}(s)=\left\|\nabla u_{s}(0)\right\|_{s}^{s}$. Thus, we find the same result stated by Lindqvist in [19]. Indeed, the inequality (3.6) becomes

$$
p\left(\lambda_{1}(p)\right)^{1 / p} \leq s\left(\lambda_{1}(s)\right)^{1 / s}
$$

Corollary 3.5 For any $\lambda \in \mathbb{R}$, we have

$$
\limsup _{s \rightarrow p_{-}} \mu_{s}(\lambda) \leq \mu_{p}(\lambda) \leq \liminf _{s \rightarrow p_{+}} \mu_{s}(\lambda)
$$

Proof Let us $s>p$, letting $s \rightarrow p_{+}$, we find

$$
\mu_{p}(\lambda) \leq \liminf _{s \rightarrow p_{+}} \mu_{s}(\lambda)
$$

because $\lim _{s \rightarrow p_{+}} \lambda_{1}(s)=\lambda_{1}(p)>0, c f$. [19].

For the case $s \rightarrow p_{-}$, (3.6) can be written for $s<p$ as

$$
\mu_{s}(\lambda) \leq \mu_{p}(\lambda)+\left[\left(\frac{p}{s}\right)^{s}\left(\lambda_{1}(p)\right)^{\frac{p}{s}-1}-1\right]\left\|\nabla u_{p}(\lambda)\right\|_{p}^{p} .
$$

This yields

$$
\limsup _{s \rightarrow p_{-}} \mu_{s}(\lambda) \leq \mu_{p}(\lambda) .
$$

Altogether, this achieves the proof.

Remark 3.6 (i) Observe that if $\lim _{s \rightarrow p} \mu_{s}(\lambda)$ exists, then this limit is necessarily equal to $\mu_{p}(\lambda)$. Thus in this case we say that we have the stability (continuity) of the point $\left(\lambda, \mu_{p}(\lambda)\right)$ of the principal eigencurve with respect to $p$. (ii) The first inequality in (3.9) can be strict in an irregular domain and $1<p \leq N$.

Theorem 3.7 For any $\lambda \in \mathbb{R}$ and for any bounded domain having the segment property, the function $p \rightarrow \mu_{p}(\lambda)$ is continuous from $(1, \infty)$ into $\mathbb{R}$.

Proof Fix $\lambda$ in $\mathbb{R}$. Let $\left\{s_{j}\right\}_{j=1}^{j=\infty}$ be a sequence in $(1, \infty)$ such that $\lim _{j \rightarrow \infty} s_{j}=p$. We claim that $\lim _{j \rightarrow \infty} \mu_{s_{j}}(\lambda)=\mu_{p}(\lambda)$. Indeed, let $\varphi \in C_{0}^{\infty}(\Omega) \backslash\{0\}$. Therefore

$$
\mu_{s_{j}}(\lambda) \leq \frac{\|\nabla \varphi\|_{s_{j}}^{s_{j}}-\lambda \int_{\Omega} m(x)|\varphi|^{s_{j}} d x}{\|\varphi\|_{s_{j}}^{s_{j}}} .
$$

Passing to the minimum over $\varphi$, we find

$$
\limsup _{j \rightarrow \infty} \mu_{s_{j}}(\lambda) \leq \mu_{p}(\lambda)
$$

To achieve the claim, it suffices to show that

$$
\mu_{p}(\lambda) \leq \liminf _{j \rightarrow \infty} \mu_{s_{j}}(\lambda)
$$


Indeed, let $\left\{s_{k}\right\}_{k \geq 1}$ be a subsequence of $\left\{s_{j}\right\}_{j \geq 1}$ such that

$$
\lim _{k \rightarrow \infty} \mu_{s_{k}}(\lambda)=\liminf _{j \rightarrow \infty} \mu_{s_{j}}(\lambda)
$$

From the minimizing property of $\mu_{s_{j}}(\lambda)$, there exists $v_{k} \in W_{0}^{1, s_{k}}(\Omega)$ such that $v_{k} \in$ $\mathrm{U}_{s_{k}}, \int_{\Omega}\left|\nabla v_{k}\right|^{s_{k}} d x>0$ and $v_{k}$ is an eigenfunction associated to eigenpair $\left(\lambda, \mu_{s_{k}}(\lambda)\right)$. Set

$$
w_{k}=\frac{v_{k}}{\left\|\nabla v_{k}\right\|_{s_{k}}}
$$

Then $w_{k} \in W_{0}^{1, s_{k}}(\Omega)$ and

$$
\begin{gathered}
\int_{\Omega}\left|\nabla w_{k}\right|^{s_{k}} d x=1, \\
\int_{\Omega}\left|w_{k}\right|^{s_{k}} d x>0 \\
\mu_{s_{k}}(\lambda) \int_{\Omega}\left|w_{k}\right|^{s_{k}} d x=1-\lambda \int_{\Omega} m(x)\left|w_{k}\right|^{s_{k}} d x .
\end{gathered}
$$

On the other hand, we have $s_{k} \rightarrow p$, as $k \rightarrow \infty$. Thus for every $\varepsilon>0$ there exists $k_{\varepsilon} \in \mathbb{N}^{*}$ such that

$$
p-\varepsilon<s_{k}<p+\varepsilon, \quad \text { when } k \geq k_{\varepsilon} .
$$

We have $p>1$, let $\varepsilon_{1}>0$ such that $p-\varepsilon_{1}>1$. Hence there exists $k_{0} \in \mathbb{N}^{*}$ such that

$$
1<p-\varepsilon_{1}<s_{k}<p+\varepsilon_{1}, \quad \text { when } k \geq k_{0} .
$$

Now let $\varepsilon_{2}>0$ small enough so that $0<\varepsilon_{2}<\sqrt{N^{2}+p^{2}}-N$. Fix $\varepsilon_{0}>0$ such that $0<\varepsilon_{0} \leq \min \left(\varepsilon_{1}, \varepsilon_{2}\right)$. Then each $\varepsilon \in\left(0, \varepsilon_{0}\right)$ satisfies

$$
1<p-\varepsilon<s_{k}<p+\varepsilon<(p-\varepsilon)^{*}, \quad \text { when } k \geq k_{0},
$$

where for any $q \in(1, \infty), q^{*}=\frac{N q}{N-q}$ if $q<N$ and $q^{*}=\infty$ if $q>N$. Note that we are interested in $k$ large enough since we have $j \rightarrow+\infty$

From (3.11) and Hölder's inequality, we obtain

$$
\left\|\nabla w_{k}\right\|_{p-\varepsilon} \leq|\Omega|^{\frac{s_{k}-p+\varepsilon}{s_{k}}}, \quad \text { when } 0<\varepsilon<\varepsilon_{0} .
$$

Hence $\left\{w_{k}\right\}_{k \geq 1}$ is a bounded sequence in $W_{0}^{1, p-\varepsilon}(\Omega)$, whenever $0<\varepsilon<\varepsilon_{0}$. Thus there is a subsequence noted also $\left\{w_{k}\right\}_{k \geq 1}$ and a function $u \in W_{0}^{1, p-\varepsilon}(\Omega)$ such that $w_{k} \rightarrow u$ (weakly) in $W_{0}^{1, p-\varepsilon}(\Omega)$, as $k \rightarrow \infty$. By compactness, we have $w_{k} \rightarrow u$ in $L^{p+\varepsilon}(\Omega)$ in view of (3.14). Moreover, we can assume that $w_{k} \rightarrow u$ a.e. on $\Omega$ as $k \rightarrow \infty$ (for a subsequence if necessary). In particular $u \in L^{p}(\Omega)$ and is independent of $\varepsilon$. Moreover, by the Dominated Convergence Theorem and using Remark 2.6 and Lemma 2.5, we obtain

$$
\int_{\Omega}\left|w_{k}\right|^{s_{k}} d x \rightarrow \int_{\Omega}|u|^{p} d x \text { and } \int_{\Omega} m(x)\left|w_{k}\right|^{s_{k}} d x \rightarrow \int_{\Omega} m(x)|u|^{p} d x
$$


as $k \rightarrow \infty$. Letting $k \rightarrow \infty$ in (3.13), we deduce that

$$
\left(\lim _{k \rightarrow \infty} \mu_{s_{k}}(\lambda)\right) \int_{\Omega}|u|^{p} d x=1-\lambda \int_{\Omega} m(x)|u|^{p} d x
$$

On the other hand, the weak lower semicontinuity of the norm yields

$$
\|\nabla u\|_{p-\varepsilon} \leq|\Omega|^{\frac{\varepsilon}{p}}
$$

when $0<\varepsilon<\varepsilon_{0}$. Fatou's lemma implies that

$$
\int_{\Omega}\|\nabla u\|^{p} d x \leq 1
$$

It follows that $u \in W^{1, p}(\Omega)$. (3.16) yields that $\int_{\Omega}|u|^{p} d x>0$. From this, (3.16) and (3.18), we find

$$
\left(\lim _{k \rightarrow \infty} \mu_{s_{k}}(\lambda)\right) \int_{\Omega}|u|^{p} d x \geq \int_{\Omega}\|\nabla u\|^{p} d x-\lambda \int_{\Omega} m(x)|u|^{p} d x .
$$

We have $u \in W^{1, p}(\Omega) \cap W_{0}^{1, p-\varepsilon}(\Omega)$, then from Theorem 2.2, $u \in W_{0}^{1, p}(\Omega)$. Consequently, $u$ is an admissible function in the definition of $\mu_{p}(\lambda)$. Hence

$$
\mu_{p}(\lambda) \int_{\Omega}|u|^{p} d x \leq \int_{\Omega}\|\nabla u\|^{p} d x-\lambda \int_{\Omega} m(x)|u|^{p} d x
$$

Finally, (3.19) and (3.20) imply that

$$
\lim _{k \rightarrow \infty} \mu_{s_{k}}(\lambda) \geq \mu_{p}(\lambda)
$$

That is,

$$
\liminf _{j \rightarrow \infty} \mu_{s_{j}}(\lambda) \geq \mu_{p}(\lambda)
$$

(3.10) and (3.21) show that

$$
\lim _{j \rightarrow \infty} \mu_{s_{j}}(\lambda)=\mu_{p}(\lambda)
$$

This concludes the proof of the claim. The theorem follows.

Remark 3.8 For the proof of the continuity on the right, the regularity of the domain is not necessary. It suffices to use (3.10) and Corollary 3.5. In the case of an irregular bounded domain and $p<N$, we can have $\lim _{s \rightarrow p_{-}} \mu_{s}(\lambda)<\mu_{p}(\lambda)$. 


\section{Convergence Results}

Throughout this section, we assume that $\Omega$ satisfies the segment property.

Theorem 4.1 For any $\lambda \in \mathbb{R}$, we have the following:

$$
\begin{aligned}
& \lim _{s \rightarrow p_{+}} \int_{\Omega}\left|\nabla u_{s}(\lambda)-\nabla u_{p}(\lambda)\right|^{p} d x=0, \\
& \lim _{s \rightarrow p_{-}} \int_{\Omega}\left|\nabla u_{s}(\lambda)-\nabla u_{p}(\lambda)\right|^{s} d x=0,
\end{aligned}
$$

where $u_{s}(\lambda)$ denotes the principal eigenfunction associated to $\left(\lambda, \mu_{s}(\lambda)\right)$.

Let us mention that for $\lambda=0$ this result is due to Lindqvist, see [19].

Proof For $s \in(p, p+1)$, Hölder's inequality yields

$$
\int_{\Omega}\left|\nabla u_{s}(\lambda)\right|^{p} d x \leq|\Omega|^{1-\frac{p}{s}}\left(\int_{\Omega}\left|\nabla u_{s}(\lambda)\right|^{s} d x\right)^{p / s} .
$$

From Lemma 3.1, we deduce that the set $\left\{u_{s} ; s \in(p, p+1)\right\}$ is bounded in $W_{0}^{1, p}(\Omega)$. Hence there exist a sequence $\left\{s_{j}\right\}_{j}$ converging to $p_{+}$and a function $u \in W_{0}^{1, p}(\Omega)$ such that $u_{s_{j}}(\lambda) \rightarrow u$ (weakly) in $W_{0}^{1, p}(\Omega)$ as $j \rightarrow \infty$. Using the Rellich-Kondrachov Compactness Theorem, $c f$. $[1,16]$, we obtain that $u_{s_{j}}(\lambda) \rightarrow u$ in $L^{p}(\Omega)$, as $j \rightarrow \infty$. Passing to a subsequence if necessary, we can assume that $u_{s_{j}}(\lambda) \rightarrow u$ a.e. on $\Omega$. Thus $u \geq 0$ a.e. on $\Omega$. We will prove that $u \equiv u_{p}(\lambda)$. From (4.3) we obtain that

$$
\left(\int_{\Omega}\left|\nabla u_{s}(\lambda)\right|^{p} d x\right)^{\frac{s}{p}} \leq|\Omega|^{\frac{s}{p}-1}\left(\mu_{s}(\lambda)+\lambda \int_{\Omega} m(x)\left\{u_{s}(\lambda)\right\}^{s} d x\right) .
$$

The weak lower semicontinuity of the norm, the Dominated Convergence Theorem and the fact that $\lim _{s \rightarrow p_{+}} \mu_{s}(\lambda)=\mu_{p}(\lambda)$ imply that

$$
\int_{\Omega}|\nabla u|^{p} d x-\lambda \int_{\Omega} m(x) u^{p} d x \leq \mu_{p}(\lambda)
$$

On the other hand, $u \in \mathrm{U}_{p}$. Therefore, $u$ is admissible in the definition of $\mu_{p}(\lambda)$. Thus we conclude from (4.5) and the variational characterization of $\mu_{p}(\lambda)$ thatthe

$$
\mu_{p}(\lambda)=\int_{\Omega}|\nabla u|^{p} d x-\lambda \int_{\Omega} m(x) u^{p} d x
$$

Finally, by the uniqueness of the principal eigenfunction associated to the eigenpair $\left(\lambda, \mu_{p}(\lambda)\right)$, we assert that $u \equiv u_{p}(\lambda)$. Thus the limit function $u$ does not depend on the particular (sub)sequence $s_{1}, s_{2}, \ldots$ Therefore $u_{s}(\lambda) \rightarrow u_{p}(\lambda)$ at least in $L^{p}(\Omega)$, as $s \rightarrow p_{+}$. The strong convergence (4.1) can be obtained from Clarkson's inequalities in $W_{0}^{1, p}(\Omega), c f .[1]$. 
For the convergence (4.2), we process as follows. Let $\varepsilon_{0}>0$ be small enough so that for any $\varepsilon \in\left(0, \varepsilon_{0}\right)$ and each $s$ close enough to $p$, we have

$$
1<p-\varepsilon<s<p+\varepsilon<(p-\varepsilon)^{*} .
$$

Such an $\varepsilon_{0}$ exists because we suppose that as $s \rightarrow p_{-}$Hölder's inequality yields

$$
\int_{\Omega}\left|\nabla u_{s}(\lambda)\right|^{p-\varepsilon} d x \leq|\Omega|^{\frac{1-p+\varepsilon}{s}}\left(\int_{\Omega}\left|\nabla u_{s}(\lambda)\right|^{s} d x\right)^{\frac{p-\varepsilon}{s}},
$$

whenever $0<\varepsilon<\varepsilon_{0}$. We use the same argument in the proof of the stability to the left $\left(s \rightarrow p_{-}\right)$. It is not difficult to have $u_{s}(\lambda)$ converge to $u(\lambda)$ weakly in $W_{0}^{1, p-\varepsilon}(\Omega)$, strongly in $L^{p}(\Omega)$ and almost everywhere in $\Omega$, as $s \rightarrow p_{-}$, when $\varepsilon>0$ satisfying (4.6), for some $u(\lambda) \in W^{1, p}(\Omega) \cap W_{0}^{1, p-\varepsilon}(\Omega)$. By Theorem 2.2, $u(\lambda) \in W_{0}^{1, p}(\Omega)$ and by the uniqueness of the principal eigenfunction associated to the eigenpair $\left(\lambda, \mu_{p}(\lambda)\right)$, we deduce that $u \equiv u_{p}(\lambda)$.

To achieve the proof, we use Clarkson's inequalities related to uniform convexity of $W_{0}^{1, s}(\Omega)$. Consider the case $p>2$ first. Since $s \rightarrow p_{-}$, we take $s$ near to $p$ so that $2 \leq s<p$ and we use Clarkson's inequality associated to $W_{0}^{1, s}(\Omega)$. The case $1<p \leq 2$ is similar, the only change being to choose the other Clarkson inequality associated to $W_{0}^{1, s}(\Omega)$, since in this case $1<s \leq 2$. The theorem follows.

Remark 4.2 (i) The convergence (4.1) is also true for any bounded domain. (ii) In any arbitrary bounded domain, we can show that the convergence (4.2) implies stability to the left, i.e., for any $\lambda \in \mathbb{R} \lim _{s \rightarrow p_{-}} \mu_{s}(\lambda)=\mu_{p}(\lambda)$. The converse is an open question when $p<N$.

Using the regularity $C_{\text {loc }}^{1+\alpha}(\Omega)$ of the principal eigenfunctions $u_{s}(\lambda)$ and the $L^{\infty}$-estimation established in Remark 2.6, we can state the following important result generalizing [19].

Theorem 4.3 For any $\lambda \in \mathbb{R}$ and $p \in(1, \infty)$ and for any bounded domain $\Omega$, each sequence converging to $p$, contains a subsequence $s_{1}, s_{2}, \ldots, s_{j}$ such that $u_{s_{j}}(\lambda) \rightarrow u(\lambda)$ and $\nabla u_{s_{j}}(\lambda) \rightarrow \nabla u(\lambda)$, locally uniformly in $\Omega$, where $u(\lambda)$ is some function in $C^{1}(\Omega)$. Moreover, $u(\lambda)$ is a weak solution to the equation

$$
\Delta_{p} u+\lambda m(x)|u|^{p-2} u+\mu(\lambda)|u|^{p-2} u=0,
$$

where $\mu(\lambda)=\lim _{j \rightarrow+\infty} \mu_{s_{j}}(\lambda)$.

\section{Remarks}

(1) (i) The limit $\lim _{s \rightarrow p_{-}} \mu_{s}(\lambda)$ can fail to exist, because the limit function $u(\lambda)$ can depend on the particular choice of $s_{1}, s_{2}, \ldots, s_{j}$.

(ii) The limit function $u(\lambda)$ is positive. Indeed, since each $u_{s_{j}}(\lambda)>0$, we have $u(\lambda) \geq 0$. Moreover $u(\lambda) \in W^{1, p}(\Omega) \cap W_{0}^{1, p-\epsilon}(\Omega)$, for $\epsilon>0$ small enough. Applying the Maximum Principle to (4.8), (cf. [26]), we conclude that $u(\lambda)>0$ in $\Omega$. 
(iii) We know that only the first eigenfunctions are not changing signs. Thus from (ii), if $\mu(\lambda)$ is an eigenvalue associated to $\lambda$ then $\mu(\lambda)=\mu_{p}(\lambda)$, and by normalization, we have $u(\lambda) \equiv u_{p}(\lambda)$.

(2) An important point: even though for any $\varepsilon>0$ chosen sufficiently small the limit function $u(\lambda)$ is in $W^{1, p}(\Omega) \cap W_{0}^{1, p-\varepsilon}(\Omega)$, it is not always the right eigenfunction $u_{p}(\lambda)$, i.e., $u(\lambda)$ is not necessarily in $W_{0}^{1, p}(\Omega)$. Thus $u(\lambda)$ is not admissible in the definition of $\mu_{p}(\lambda)$.

(3) If $\Omega$ has the segment property as regularity, then $u(\lambda) \equiv u_{p}(\lambda), \mu(\lambda)=\mu_{p}(\lambda)$ and $\lim _{s \rightarrow p} \mu_{s}(\lambda)=\mu_{p}(\lambda)$.

Remark 4.4 (Generalization) Many results are readily extended to equations of the more general form

$$
-\Delta_{p} u+h(x)|u|^{p-2} u-\lambda m(x)|u|^{p-2} u=\mu g(x)|u|^{p-2} u
$$

$u \in W_{0}^{1, p}(\Omega)$, where $\Omega$ is a bounded domain of $\mathbb{R}^{N}, N \geq 1, h, m \in L^{\infty}(\Omega)$, with $m \not \equiv 0$ and $g \in L^{\infty}(\Omega)$ with $g \geq \varepsilon_{0}>0$ a.e. on $\Omega$, for some $\varepsilon_{0}$.

\section{References}

[1] R. Adams, Sobolev Spaces, Pure and Applied Mathematics 65, Academic Press, New York, 1975.

[2] A. Anane, Simplicité et isolation de la première valeur propre du p-Laplacien avec poids. C. R. Acad. Sci. Paris, Sér. I Math. 305(1987), no. 16, 725-728.

[3] C. Atkinson and C. R. Champion, Some-boundary value problems for the equation $\nabla \cdot\left(|\nabla \phi|^{N}\right)=0$. Quart. J. Mech. Appl. Math. 37(1984), no. 3, 401-419.

[4] C. Atkinson and C. W. Jones, Similarity solutions in some nonlinear diffusion problems and in boundary-layer flow of a pseudo plastic fluid. Quart. J. Mech. Appl. Math. 27(1974), 193-211.

[5] J. P. G. Azorero and I. P. Alonso, Existence and uniqueness for the p-Laplacian: nonlinear eigenvalues. Comm. Partial Differential Equations 12(1987), no. 12, 1389-1430.

[6] G. I. Barenblatt, On self similar notions of compressible fluids in a porous medium. Prikl. Mat. Mekh. 16(1952), 679-698 (in Russian).

[7] P. A. Binding and Y. X. Huang, The principal eigencurve for the p-Laplacian. Differential Integral Equations 8(1995), no. 2, 405-414.

[8] H. Brezis, Analyse fonctionnelle. Théorie et applications. Collection Mathématiques Appliquées pour la Maitrise. Masson, Paris, 1983.

[9] R. Courant and D. Hilbert, Methods of Mathematical Physics. Vol. 1, Interscience Publishers, New York, 1953.

[10] J. I. Diaz, Nonlinear partial differential equations and free boundaries. Vol. 1, Elliptic Equations, Research Notes in Mathematics 106, Pitman, Boston, 1985.

[11] E. Dibenedetto, $C^{1+\alpha}$-local regularity of weak solutions of degenerate elliptic equations. Nonlinear Anal. 7(1983), no. 8, 827-850.

[12] D. E. Edmunds and W. D. Evans, Spectral Theory and Differential Operators. Clarendon Press, Oxford, 1990

[13] A. El Khalil, Autour de la première courbe propre du p-Laplacien. Thèse de doctorat, Faculté des Sciences Dhar-Mahraz, Fès, 1999.

[14] A. El Khalil, P. Lindqvist, and A. Touzani, On the stability of the first eigenvalue of $A_{p} u+\lambda g(x)|u|^{p-2} u=0$ with varying p. Rend. Mat. Appl. 24(2004), no. 2, 321-336.

[15] $\longrightarrow$ On the first eigenvalue of the p-Laplacian. In: Partial Differential Equations, Lecture Notes in Pure and Applied Mathematics 229, Dekker, New York, 2002, pp. 195-205.

[16] D. Gilbarg and N. Trudinger, Elliptic Partial Differential Equations of Second Order. Grundlehren der Mathematischen Wissenschaften 224, Springer-Verlag, Berlin, 1983.

[17] P. Hess and T. Kato, On some linear and nonlinear eigenvalue problems with an indefinite weight function. Comm. Partial Differential Equations 5(1980), no. 10, 999-1030. 
[18] P. Lindqvist, On the equation $\operatorname{div}\left(|\nabla u|^{p-2} \nabla u\right)+\lambda|u|^{p-2} u=0$. Proc. Amer. Math. Soc. 109(1990), no. 1, 157-164.

[19] On nonlinear Rayleigh quotients. Potential Anal. 2(1993), no. 3, 199-218.

[20] J.-L. Lions, Quelques méthodes de résolution des problèmes aux limites non linéaires. Dunod, Paris, 1969.

[21] M. Otani and T. Teshima, On the first eigenvalue of some quasilinear elliptic equations. Proc. Japan Acad. Sci. Ser. A. Math. Sci. 64(1988), no. 1, 8-10.

[22] M. C. Pelissier and L. Reynaud, Étude d'un modèle mathématique d'écoulement de glacier. C. R. Acad. Sci. Paris Sér. A 279(1974), 531-534.

[23] J. R. Philip, n-diffusion. Austral. J. Phys. 14(1961), 1-13.

[24] R. E. Showalter, Monotone Operators in Banach Spaces and Nonlinear Partial Differential Equations. Mathematical Surveys and Monographs 49, American Mathematical Society, Providence, RI, 1997.

[25] J. Smagorinsky, General circulation experiments with the primitive equations. I: The basic experiment. Monthly Wea. Rev. 91(1963), 99-152.

[26] J. L. Vázquez, A strong maximum principle for some quasilinear elliptic equations. App. Math. Optim. 12(1984), no. 3, 191-202.

Département de Mathématiques et de Génie Industriel

École Polytechnique de Montréal

Montréal, QC

e-mail: abdelouahed.el-khalil@polymtl.ca
Department of Mathematics Faculty of Sciences Dhar-Mahraz

P.O. Box 1796 Atlas

Fez 30000

Morocco

e-mail:manouni@hotmail.com

m_ouanan@hotmail.com 